\title{
Non-Centralized Navigation for Source Localization by Cooperative UAVs
}

\author{
Anna Guerra ${ }^{\dagger} \quad$ Davide Dardari ${ }^{\dagger} \quad$ Petar M. Djurić * \\ * DEI-CNIT, University of Bologna, 47521, Cesena, Italy. \\ $\dagger$ ECE, Stony Brook University, Stony Brook, NY 11794, USA. \\ E-mail: \{anna.guerra3, davide.dardari\}@unibo.it; petar.djuric@stonybrook.edu
}

\begin{abstract}
In this paper, we propose a distributed solution to the navigation of a population of unmanned aerial vehicles (UAVs) to best localize a static source. The network is considered heterogeneous with UAVs equipped with received signal strength (RSS) sensors from which it is possible to estimate the distance from the source and/or the direction of arrival through adhoc rotations. This diversity in gathering and processing RSS measurements mitigates the loss of localization accuracy due to the adoption of low-complexity sensors. The UAVs plan their trajectories on-the-fly and in a distributed fashion. The collected data are disseminated through the network via multi-hops, therefore being subject to latency. Since not all the paths are equal in terms of information gathering rewards, the motion planning is formulated as a minimization of the uncertainty of the source position under UAV kinematic and anti-collision constraints and performed by 3D non-linear programming. The proposed analysis takes into account non-line-of-sight (NLOS) channel conditions as well as measurement age caused by the latency constraints in communication.
\end{abstract}

Index Terms-Unmanned aerial vehicles, RSS localization, UAV navigation, Information gathering.

\section{INTRODUCTION}

In recent years, unmanned aerial vehicles (UAVs) have become more and more autonomous and small, increasing the possibility of creating swarms of small flying drones able to mimic the collaborative behavior of insects [1], [2]. The main motivation underlying the swarming interest is that team strategies can boost the flexibility and robustness of current wireless sensor networks. In fact, UAVs are expected to locally sense and interact with the environment and collaborate with each other. Moreover, the redundancy of the information coming through the network permits to lower the possibility of deterioration from the loss or malfunctioning of a single node. All these characteristics are exploited to enable a large number of applications [3]- [6].

In this context, the optimization of UAV trajectories has been the subject of numerous research studies [7]-[9]. Among other approaches, information-seeking optimal control (i.e., strategies driven by Shannon or Fisher information measures) has been extensively investigated for localization and tracking applications [10], [11]. For instance, in [12], the problem of an off-line (pre-mission) path design for best source location

This work has received funding from the European Union's Horizon 2020 research and innovation programme under the Marie Sklodowska-Curie project AirSens (grant no. 793581). P. M. D. thanks the support of the NSF under Award CCF-1618999.

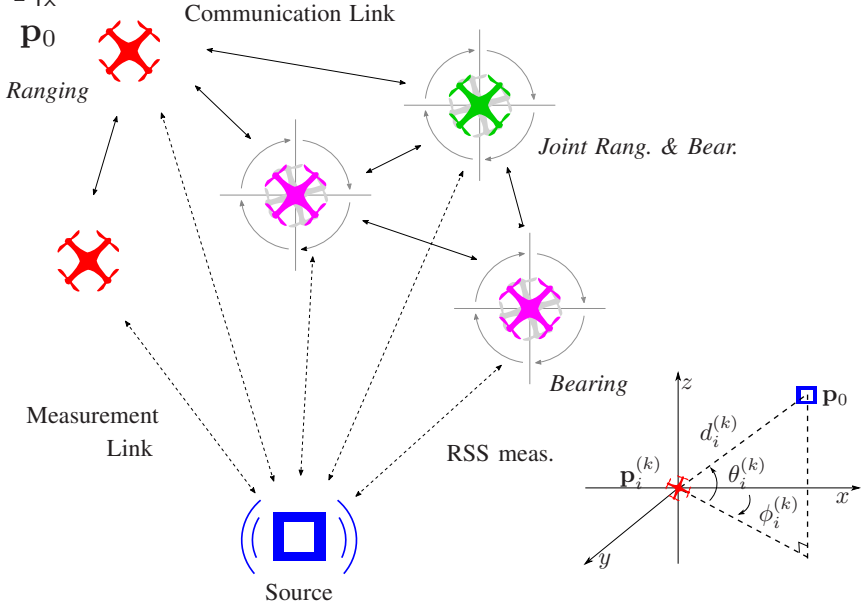

Fig. 1. A UAV network, where different groups of UAVs acquire RSS measurements. On the left, starting from the RSS information, the red UAVs estimate ranging-only, the magenta bearing-only, and the green both ranging and bearing parameters. From these estimates, they navigate to best localize the source at $\mathbf{p}_{0}$. On the right, the coordinate system is depicted.

using two mobile sensors is addressed. A real-time approach is proposed in [13], where multiple UAVs acquire differential received signal strength (RSS) measurements for rangingbased tracking. In [14], the motion planning is interpreted as an adaptive sensing strategy and results show the superiority of the D-optimality approach over other solutions.

In this paper, we adopt the concept of information-seeking control and we propose a distributed navigation scheme for a network of UAVs with different sensing and processing roles, as represented in Fig. 1. Differently from the state-of-the art, a group of UAVs might infer ranging-only information while another bearing-only or both, and the obtained information is disseminated through the network via multi-hops, and hence is subjected to latency. In particular, by assuming that the UAVs know their positions thanks to on-board GPS modules, their goal is to navigate an outdoor environment for best localizing a source. For this purpose, the UAVs rely on measurements coming from on-board RSS sensors, thus avoiding the use of antenna arrays, whose size and cost could be incompatible with the integration in UAVs.

The UAV-source distance can be inferred from the RSS measurements and using a path-loss model [15]. On the other hand, the direction-of-arrival (DOA) is associated with the 
angle from which the maximum RSS is experienced. More specifically, UAV rotations might be exploited to point the sensor antenna in different angular directions and to form a RSS pattern after each rotation as in [16]. Nevertheless, the time needed to search for the bearing direction and perform a complete rotation prevents drones from taking navigation decisions quickly. Hence, we suppose that not all the UAVs collect bearing measurements, opting instead for a network with heterogeneous drones. The fact that not all UAVs of the network have the possibility to estimate both ranging and bearing data might decrease the localization performance, but it also helps in reducing the time-to-navigation by avoiding UAV rotations.

Finally, since the paths are different from an information gathering point-of-view, the problem is formulated as a 3D optimization where the function to be minimized depends on the Fisher Information Matrix (FIM), constrained by the UAV kinematics and anti-collision requirements.

\section{Problem Statement}

\section{A. UAV Dynamic Model}

We consider a network of $N$ UAVs acting as mobile reference nodes (i.e., with known positions, for instance from GPS) whose objective is to navigate through an environment to optimize the accuracy in localizing a source in $\mathbf{p}_{0}=\left[x_{0}, y_{0}, z_{0}\right]^{\mathrm{T}}$.

At each time instant, the next position of the $i$ th UAV is given by $\mathbf{p}_{i}^{(k+1)}=\varphi\left(\mathbf{p}_{i}^{(k)}, \mathbf{u}_{i}^{(k+1)}\right)$, where $\varphi(\cdot)$ is the transition function, $\mathbf{p}_{i}^{(k)}=\left[x_{i}^{(k)}, y_{i}^{(k)}, z_{i}^{(k)}\right]^{\mathrm{T}}$ is the position of the $i$ th UAV at time instant $k$ and $\mathbf{u}_{i}^{(k)}=\left[u_{\mathrm{x}, i}^{(k)}, u_{\mathrm{y}, i}^{(k)}, u_{\mathrm{z}, i}^{(k)}\right]^{\mathrm{T}}=$ $g\left(v_{i}^{(k)}, \Psi_{i}^{(k)}, \Theta_{i}^{(k)}\right)$ is the control signal computed by the $i$ th UAV on its own that enables as accurate localization of the source as possible [8]. The speed, the heading and the tilt angles are indicated with $v_{i}^{(k)}, \Psi_{i}^{(k)}$, and $\Theta_{i}^{(k)}$, respectively. In particular, the update for the position is given by

$$
\begin{aligned}
& {\left[\begin{array}{l}
x_{i}^{(k+1)} \\
y_{i}^{(k+1)} \\
z_{i}^{(k+1)}
\end{array}\right]=\left[\begin{array}{c}
x_{i}^{(k)}+u_{\mathrm{x}, i}^{(k+1)} \\
y_{i}^{(k)}+u_{\mathrm{y}, i}^{(k+1)} \\
z_{i}^{(k)}+u_{\mathrm{z}, i}^{(k+1)}
\end{array}\right]=} \\
& =\left[\begin{array}{c}
x_{i}^{(k)}+\left(v_{i}^{(k+1)} \cdot \Delta t\right) \cos \left(\Psi_{i}^{(k+1)}\right) \cos \left(\Theta_{i}^{(k+1)}\right) \\
y_{i}^{(k)}+\left(v_{i}^{(k+1)} \cdot \Delta t\right) \sin \left(\Psi_{i}^{(k+1)}\right) \cos \left(\Theta_{i}^{(k+1)}\right) \\
z_{i}^{(k)}+\left(v_{i}^{(k+1)} \cdot \Delta t\right) \sin \left(\Theta_{i}^{(k+1)}\right)
\end{array}\right] .
\end{aligned}
$$

To make the model more realistic, three constraints are added to impose the minimum and maximum speed and a maximum turn rate in both azimuthal and elevation planes [11], i.e.,

$$
\left\{\begin{array}{l}
v_{\min } \leq\left\|\mathbf{u}_{i}^{(k+1)}\right\| / \Delta t \leq v_{\max }, \\
\left|\Psi_{i}^{(k+1)}-\Psi_{i}^{(k)}\right| \leq \phi_{\max }, \\
\left|\Theta_{i}^{(k+1)}-\Theta_{i}^{(k)}\right| \leq \theta_{\max },
\end{array}\right.
$$

with $\Delta t$ being the time step, $v_{\min }$ and $v_{\max }$ the minimum and maximum UAV speeds, and $\phi_{\max }$ and $\theta_{\max }$ the turn rate limits. The geometry of the system is depicted in Fig. 1 right.

\section{B. Observation Model}

The UAVs obtain RSS measurements, and from the acquired data, they extract ranging and/or bearing information from which the position of the source is estimated (two-step localization). Since each UAV can process its measurements in a different way according to its capabilities, we indicate with $\mathcal{N}_{\mathrm{r}}$ the set of UAVs that obtains ranging-only estimates, with $\mathcal{N}_{\mathrm{b}}$ the set that finds bearing-only estimates, and with $\mathcal{N}_{\mathrm{j}}$ the set with both types of estimates. The network composed of all the heterogeneous UAVs is denoted by $\mathcal{N}=\mathcal{N}_{\mathrm{r}} \cup \mathcal{N}_{\mathrm{b}} \cup \mathcal{N}_{\mathrm{j}}$.

The ranging/bearing estimation errors and the positions of the UAVs are shared through the network via multi-hops. Each node can directly communicate with its neighbors within a radius of length $d_{\text {hop }}$, while for greater distances, the information is delayed by $h_{i j}^{(k)}$ time slots, equal to the number of hops between the $i$ th and $j$ th UAV at instant $k$. Moreover, we assume that the connectivity is always guaranteed; as soon as a new measurement becomes available, it is recorded in an internal memory buffer and, thus, if, by chance, the UAVs get isolated, it is possible to rely on the latest saved information.

After this exchange, the data vector collected by the $i$ th UAV at time instant $k$ is $\mathbf{z}_{i}^{(k)}=\left[\ldots, \tilde{\mathbf{z}}_{j}^{\left(\ell_{k}\right)}, \ldots\right]^{\mathrm{T}}$, where $\tilde{\mathbf{z}}_{j}^{\left(\ell_{k}\right)}$ is the estimate inferred by the $j$ th UAV and arriving at the $i$ th node with a delay of $\ell_{k}=k-h_{i j}^{(k)}+1$. If $h_{i j}^{(k)}$ exceeds the maximum number of allowed hops (i.e., $h_{\max }$ ), the UAVs refer to the last saved information. More specifically, the generic estimate is

$\tilde{\mathbf{z}}_{j}^{\left(\ell_{k}\right)}= \begin{cases}\hat{d}_{j}^{\left(\ell_{k}\right)}=d_{j}^{\left(\ell_{k}\right)}+n_{\mathrm{r}, j}^{\left(\ell_{k}\right)}, & j \in \mathcal{N}_{\mathrm{r}}, \\ \hat{\alpha}_{j}^{\left(\ell_{k}\right)}=p_{j}^{\left(\ell_{k}\right)}\left(\alpha_{j}^{\left(\ell_{k}\right)}+n_{\mathrm{b}, j}^{\left(\ell_{k}\right)}\right)+\bar{p}_{j}^{\left(\ell_{k}\right)} \omega_{j}^{\left(\ell_{k}\right)}, & j \in \mathcal{N}_{\mathrm{b}}, \\ \left.\hat{d}_{j}^{\left(\ell_{k}\right)}, \hat{\alpha}_{j}^{\left(\ell_{k}\right)}\right]^{\mathrm{T}}, & j \in \mathcal{N}_{\mathbf{j}},\end{cases}$

with $d_{j}^{\left(\ell_{k}\right)}, \alpha_{j}^{\left(\ell_{k}\right)}=\left(\phi_{j}^{\left(\ell_{k}\right)}, \theta_{j}^{\left(\ell_{k}\right)}\right)$ being the actual distance, azimuth and elevation angle between the $j$ th UAV and the source, and $n_{\mathrm{r}, j}^{\left(\ell_{k}\right)} \sim \mathcal{N}\left(0,\left(\sigma_{\mathrm{r}, j}^{\left(\ell_{k}\right)}\right)^{2}\right), n_{\mathrm{b}, j}^{\left(\ell_{k}\right)} \sim \mathcal{N}\left(0,\left(\sigma_{\mathrm{b}, j}^{\left(\ell_{k}\right)}\right)^{2}\right)$ representing their estimation errors, respectively. The symbol $p_{j}^{\left(\ell_{k}\right)}$ is a deterministic binary variable indicating the presence or absence of non line-of-sight (NLOS), $\bar{p}_{j}^{\left(\ell_{k}\right)}=1-p_{j}^{\left(\ell_{k}\right)}$, and $\omega_{j}^{\left(\ell_{k}\right)}$ is an outlier term due to multipath components [16]. The RSS-based ranging variance can be modeled, according to the Cramér-Rao lower bound (CRLB) [17], as

$$
\left(\sigma_{\mathrm{r}, j}^{\left(\ell_{k}\right)}\right)^{2}=\left(\frac{\ln 10}{10} \cdot \frac{\sigma_{\mathrm{sh}}}{\gamma}\right)^{2}\left(d_{j}^{\left(\ell_{k}\right)}\right)^{\gamma}=\sigma_{\mathrm{r}, 0}^{2}\left(d_{j}^{\left(\ell_{k}\right)}\right)^{\gamma},
$$

where $\sigma_{r, 0}$ is the ranging standard deviation (std) at the reference distance $\left(d_{0}=1 \mathrm{~m}\right), \gamma$ is the path-loss exponent and $\sigma_{\mathrm{sh}}$ is the shadowing std 1 Note that the model in (4) is valid for either LOS and NLOS settings with a proper choice of the channel parameters, i.e., of the ratio $\sigma_{\mathrm{sh}} / \gamma$. The bearing noise variance is considered constant, i.e., $\left(\sigma_{\mathrm{b}, j}^{\left(\ell_{k}\right)}\right)^{2}=\sigma_{\mathrm{b}, 0}^{2}$.

\footnotetext{
${ }^{1}$ The source transmit power and the channel parameters, i.e., $\sigma_{\text {sh }}$ and $\gamma$, are supposed to be known. For RSS localization in presence of unknown channel parameters, we refer the reader to [18].
} 
Starting from the collected measurements and the knowledge of the other UAV positions, each UAV estimates the position of the source at time instant $k$, i.e., $\hat{\mathbf{p}}_{0 i}^{(k)}$ [15]2

\section{UAV Navigation}

Once the source position has been estimated, the UAVs plan their trajectory based on a metric capturing the quality of the localization process. One possible solution is based on minimizing the root mean squared error (RMSE) of the source position estimate. However, the RMSE is strictly related to the adopted estimator and requires the knowledge of the actual source position (that is the parameter to be estimated) [19], whereas the trajectory planner should be valid for any estimator, and agnostic with respect to the actual source position. To meet the requirement of invariance over estimators, we chose the following cost functions [10]:

$$
\mathcal{C}\left(\mathbf{q}_{i}^{(k)}\right)= \begin{cases}-\ln \operatorname{det}\left(\mathbf{J}\left(\hat{\mathbf{p}}_{0 i}^{(k)} ; \mathbf{q}_{i}^{(k)}\right)\right), & \text { D-Optimality } \\ \operatorname{tr}\left(\mathbf{J}^{-1}\left(\hat{\mathbf{p}}_{0 i}^{(k)} ; \mathbf{q}_{i}^{(k)}\right)\right), & \text { A-Optimality }\end{cases}
$$

where $\mathbf{q}_{i}^{(k)}=\left[\ldots, \mathbf{p}_{j}^{\left(\ell_{k}\right)}, \ldots\right]^{\mathrm{T}}$ contains the positions of the UAVs as known by the $i$ th $\mathrm{UAV}, \operatorname{tr}(\cdot)$ and $\operatorname{det}(\cdot)$ are the trace and determinant operators, respectively, and $\mathbf{J}\left(\hat{\mathbf{p}}_{0 i}^{(k)} ; \mathbf{q}_{i}^{(k)}\right)$ is the FIM of the source location as a function of the current and previous UAV locations, and evaluated on the estimated source position. Note that $\mathbf{q}_{i}^{(k)}$ can make the cost function dependent on the previous (non-updated) locations of the drones.

Consequently, the control law at time instant $k$ at the $i$ th $\mathrm{UAV}$ is the solution of the following minimization problem:

$$
\begin{aligned}
\left(\mathbf{q}_{i}^{(k+1)}\right)^{\star}=\underset{\mathbf{q}_{i}^{(k+1)} \in \mathbb{R}^{2}}{\operatorname{argmin}} & \mathcal{C}\left(\mathbf{q}_{i}^{(k+1)}\right) \\
\text { subject to } \quad & d_{i j}^{(k)} \geq d_{\mathrm{U}}^{*}, d_{i}^{(k)} \geq d_{\mathrm{S}}^{*}, \\
& \mathcal{T}_{i} \cap \mathcal{O}=\varnothing \\
& v_{\min } \leq\left\|\mathbf{u}_{i}^{(k+1)}\right\| / \Delta t \leq v_{\max }, \\
& \left|\Psi_{i}^{(k+1)}-\Psi_{i}^{(k)}\right| \leq \phi_{\max }, \\
& \left|\Theta_{i}^{(k+1)}-\Theta_{i}^{(k)}\right| \leq \theta_{\max }, \\
& z_{\min } \leq z_{i}^{(k)} \leq z_{\max },
\end{aligned}
$$

for $i=1, \ldots, N$, and where $d_{i j}^{(k)}$ is the inter-UAV distance, $d_{\mathrm{U}}^{*}$ is the anti-collision safety distance among UAVs, $d_{\mathrm{S}}^{*}$ is the safety distance with respect to the source, $\mathcal{T}_{i}$ is the set of feasible position points of the trajectory of the $i$ th UAV, and $\mathcal{O}$ is the set of obstacles present in the environment from which the UAVs should keep a safety distance equal to $d_{\mathrm{O}}^{*}$. The last constraints impose a maximum value on the UAV turning rates and a bounding box for the flight altitude.

With (6), the drones search in a fully distributed way for the optimal UAV formation that minimizes the Fisher informationdriven function at the next time instant.

\footnotetext{
${ }^{2}$ In this paper, we focus on the path planning aspects and we consider the position estimates given as an input for the navigation algorithm.
}

Then, recalling the transition model (1), the control signal of the $i$ th UAV that satisfies (6) is given by $\mathbf{u}_{i}^{(k+1)}=$ $\left[\left(\mathbf{q}_{i}^{(k+1)}\right)^{\star}\right]_{i}-\mathbf{p}_{i}^{(k)}$, where $[\cdot]_{i}$ is an operator that picks the $i$ th entry of the optimal formation in (6), i.e., $\left(\mathbf{p}_{i}^{(k+1)}\right)^{\star}$.

\section{Cost Function DERIVATION}

Here we derive the cost function in (6) for both the A- and D-optimality cases. Firstly, we recall the FIM definition [20]

$$
\mathbf{J}\left(\mathbf{p}_{0} ; \mathbf{q}_{i}^{(k)}\right)=\mathbb{E}\left\{\left[\nabla_{\mathbf{p}_{0}} \Lambda\left(\mathbf{z}_{i}^{(k)} \mid \mathbf{p}_{0}\right)\right]\left[\nabla_{\mathbf{p}_{0}} \Lambda\left(\mathbf{z}_{i}^{(k)} \mid \mathbf{p}_{0}\right)\right]^{\mathrm{T}}\right\}
$$

with $\Lambda\left(\mathbf{z}_{i}^{(k)} \mid \mathbf{p}_{0}\right)=\ln f\left(\mathbf{z}_{i}^{(k)} \mid \mathbf{p}_{0}\right)$ being the log-likelihood function. Assuming the independence between estimates, we can write

$$
\begin{aligned}
\Lambda\left(\mathbf{z}_{i}^{(k)} \mid \mathbf{p}_{0}\right) & =\sum_{j \in \mathcal{N}} \kappa_{j} \ln f\left(\hat{d}_{j}^{\left(\ell_{k}\right)} \mid \mathbf{p}_{0}\right)+ \\
& +\beta_{j}\left(\ln f\left(\hat{\theta}_{j}^{\left(\ell_{k}\right)} \mid \mathbf{p}_{0}\right)+\ln f\left(\hat{\phi}_{j}^{\left(\ell_{k}\right)} \mid \mathbf{p}_{0}\right)\right)
\end{aligned}
$$

with $\kappa_{j}=\{0,1\}$ and $\beta_{j}=\{0,1\}$ being equal to 1 if the $j$ th UAV can estimate the ranging and/or bearing information. Following the same steps of [20], it is possible to find

$$
\begin{aligned}
& \mathbf{J}\left(\mathbf{p}_{0} ; \mathbf{q}_{i}^{(k)}\right)=\left[\begin{array}{ccc}
J_{\mathrm{xx}, i}^{(k)} & J_{\mathrm{xy}, i}^{(k)} & J_{\mathrm{xz}, i}^{(k)} \\
J_{\mathrm{xy}, i}^{(k)} & J_{\mathrm{yy}, i}^{(k)} & J_{\mathrm{yz}, i}^{(k)} \\
J_{\mathrm{zx}, i}^{(k)} & J_{\mathrm{zy}, i}^{(k)} & J_{\mathrm{zz}, i}^{(k)}
\end{array}\right] \\
& =\sum_{j \in \mathcal{N}} \kappa_{j} A_{\mathrm{r}, j}^{\left(\ell_{k}\right)} \mathbf{G}_{\mathrm{r}, j}^{\left(\ell_{k}\right)}+\beta_{j} p_{j}^{\left(\ell_{k}\right)} A_{\mathrm{b}, j}^{\left(\ell_{k}\right)}\left(\mathbf{G}_{\mathrm{az}, j}^{\left(\ell_{k}\right)}+\mathbf{G}_{\mathrm{el}, j}^{\left(\ell_{k}\right)}\right),
\end{aligned}
$$

with the subscripts $\mathrm{x}, \mathrm{y}$, and $\mathrm{z}$ indicating the Cartesian position coordinates and where

$$
\begin{aligned}
\mathbf{G}_{\mathrm{r}, j}^{\left(\ell_{k}\right)}\left(\phi_{j}^{\left(\ell_{k}\right)}, \theta_{j}^{\left(\ell_{k}\right)}\right) & =\mathbf{a}_{j}^{\left(\ell_{k}\right)}\left(\mathbf{a}_{j}^{\left(\ell_{k}\right)}\right)^{\mathrm{T}}, \\
\mathbf{G}_{\mathrm{az}, j}^{\left(\ell_{k}\right)}\left(\phi_{j}^{\left(\ell_{k}\right)}, \theta_{j}^{\left(\ell_{k}\right)}\right) & =\frac{\mathbf{G}_{\mathrm{r}, j}^{\left(\ell_{k}\right)}\left(\phi_{j}^{\left(\ell_{k}\right)}+\pi / 2,0\right)}{\left(d_{j}^{\left(\ell_{k}\right)} \cos \left(\theta_{j}^{\left(\ell_{k}\right)}\right)\right)^{2}}, \\
\mathbf{G}_{\mathrm{el}, j}^{\left(\ell_{k}\right)}\left(\phi_{j}^{\left(\ell_{k}\right)}, \theta_{j}^{\left(\ell_{k}\right)}\right) & =\frac{\mathbf{G}_{\mathrm{r}, j}^{\left(\ell_{k}\right)}\left(\phi_{j}^{\left(\ell_{k}\right)},-\theta_{j}^{\left(\ell_{k}\right)}-\pi / 2\right)}{\left(d_{j}^{\left(\ell_{k}\right)}\right)^{2}},
\end{aligned}
$$

with $\mathbf{a}_{j}^{\left(\ell_{k}\right)}$ being the direction vector given by

$$
\mathbf{a}_{j}^{\left(\ell_{k}\right)}=\left[\begin{array}{cc}
\cos \left(\phi_{j}^{\left(\ell_{k}\right)}\right) & \cos \left(\theta_{j}^{\left(\ell_{k}\right)}\right) \\
\sin \left(\phi_{j}^{\left(\ell_{k}\right)}\right) & \cos \left(\theta_{j}^{\left(\ell_{k}\right)}\right) \\
\sin \left(\theta_{j}^{\left(\ell_{k}\right)}\right) &
\end{array}\right] .
$$


Finally, the coefficients $A_{\mathrm{r}, j}^{\left(\ell_{k}\right)}$ and $A_{\mathrm{b}, j}^{\left(\ell_{k}\right)}$ depend on the measurement noise variances as 3

$$
\left\{\begin{array}{l}
A_{\mathrm{r}, j}^{\left(\ell_{k}\right)}=\left(1 /\left(\sigma_{\mathrm{r}, j}^{\left(\ell_{k}\right)}\right)^{2}\right)\left(1+2 \frac{\gamma^{2} \sigma_{\mathrm{r}, 0}^{2}\left(d_{j}^{\left(\ell_{k}\right)}\right)^{\gamma}}{4\left(d_{j}^{\left(\ell_{k}\right)}\right)^{2}}\right) \\
A_{\mathrm{b}, j}^{\left(\ell_{k}\right)}=1 / \sigma_{\mathrm{b}, 0}^{2}
\end{array} .\right.
$$

Starting from (9), the A- and D-optimality criteria can be simply derived as in (5) where, instead of using the actual source position that is not available, the UAVs consider their estimates, i.e., $\hat{\mathbf{p}}_{0 i}^{(k)}$. More specifically, we have

$\mathcal{C}\left(\mathbf{q}_{i}^{(k)}\right)=$

$\begin{cases}-\ln \left(J_{\mathrm{xx}, i}^{(k)} C_{\mathrm{xx}, i}^{(k)}+J_{\mathrm{xy}, i}^{(k)} C_{\mathrm{yx}, i}^{(k)}+J_{\mathrm{xz}, i}^{(k)} C_{\mathrm{zx}, i}^{(k)}\right), & \text { D-Optimality } \\ \frac{C_{\mathrm{x} x, i}^{(k)}+C_{\mathrm{yy}, i}^{(k)}+C_{\mathrm{zz}, i}^{(k)}}{J_{\mathrm{xx}, i}^{(k)} C_{\mathrm{xx}, i}^{(k)}+J_{\mathrm{xy}, i}^{(k)} C_{\mathrm{yx}, i}^{(k)}+J_{\mathrm{xz}, i}^{(k)} C_{\mathrm{zx}, i}^{(k)}}, & \text { A-Optimality }\end{cases}$

with the cofactors of the FIM given by $C_{\mathrm{xx}}=J_{\mathrm{yy}} J_{\mathrm{zz}}-\left(J_{\mathrm{yz}}\right)^{2}$, $C_{\mathrm{yy}}=J_{\mathrm{xx}} J_{\mathrm{zz}}-\left(J_{\mathrm{xz}}\right)^{2}, C_{\mathrm{yx}}=J_{\mathrm{yz}} J_{\mathrm{xz}}-J_{\mathrm{xy}} J_{\mathrm{zz}}, C_{\mathrm{zx}}=J_{\mathrm{xy}} J_{\mathrm{yz}}-J_{\mathrm{yy}} J_{\mathrm{xz}}$, and $C_{\mathrm{zz}}=J_{\mathrm{xx}} J_{\mathrm{yy}}-\left(J_{\mathrm{xy}}\right)^{2}$.

\section{CONTROL LaW}

The constrained minimization problem in (6) can be solved using the projection gradient method [21]

$$
\mathbf{u}_{i}^{(k+1)}=-\xi \mathbf{P} \nabla_{\mathbf{p}_{i}^{(k)}} \mathcal{C}\left(\mathbf{q}_{i}^{(k)}\right)-\mathbf{N}\left(\mathbf{N}^{\mathrm{T}} \mathbf{N}\right)^{-1} \mathbf{g},
$$

where $\xi$ represents the spatial step, $\nabla_{\mathbf{p}_{i}^{(k)}}(\cdot)$ is the gradient operator with respect to the UAV positions which, taken with the negative sign, represents the direction of decrease of the cost function. The projection matrix is denoted with $\mathbf{P}=\mathbf{I}-\mathbf{N}\left(\mathbf{N}^{\mathrm{T}} \mathbf{N}\right)^{-1} \mathbf{N}^{\mathrm{T}}$ with $\mathbf{I}$ being the identity matrix and $\mathbf{N}=\left(\nabla_{\mathbf{p}_{i}^{(k)}} \mathbf{g}\right)$ being the gradient of the constraints in $\mathbf{g}=\left[\begin{array}{lll}\mathbf{g}_{1} & \mathbf{g}_{2} & \mathbf{g}_{3}\end{array}\right]$, where

$$
\begin{aligned}
& \mathbf{g}_{1}=\mathbf{d}_{\mathrm{U}}-d_{\mathrm{U}}^{*}, \quad \mathbf{d}_{\mathrm{U}}=\left\{d_{i j}^{(k)}: d_{i j}^{(k)}<d_{\mathrm{U}}^{*}\right\}, \\
& \mathbf{g}_{2}=\mathbf{d}_{\mathrm{S}}-d_{\mathrm{S}}^{*}, \quad \mathbf{d}_{\mathrm{S}}=\left\{d_{i}^{(k)}: d_{i}^{(k)}<d_{\mathrm{S}}^{*}\right\}, \\
& \mathbf{g}_{3}=\mathbf{d}_{\mathrm{O}}-d_{\mathrm{O}}^{*},
\end{aligned}
$$

with $d_{i, \mathrm{O}}^{(k)}$ being the minimum distance between the $i$ th drone and its closest obstacle. Finally, we impose the UAV speed, altitude and the maximum turning rates by considering the last four constraints of (6).

\section{Case Study}

In this section, we analyze the evolution of the localization accuracy in relation to the UAV dynamics and sensing capabilities for a 3D scenario plotted in Fig. 2 .

The minimum and maximum speed were set to $v_{\min }=$ $0.5 \mathrm{~m} / \mathrm{step}$ and $v_{\max }=1 \mathrm{~m} / \mathrm{step}$, respectively, while the maximum turn rates per unit step to $\phi_{\max }=\theta_{\max }=50^{\circ}$. The estimation errors were $\sigma_{\mathrm{sh}} / \gamma=1.7$ for $\operatorname{LOS}, \sigma_{\mathrm{sh}} / \gamma=3.2$

\footnotetext{
${ }^{3}$ Thanks to the possibility to discriminate LOS/NLOS situations, we assume that the UAVs exactly know the values of the coefficients in 14.
}
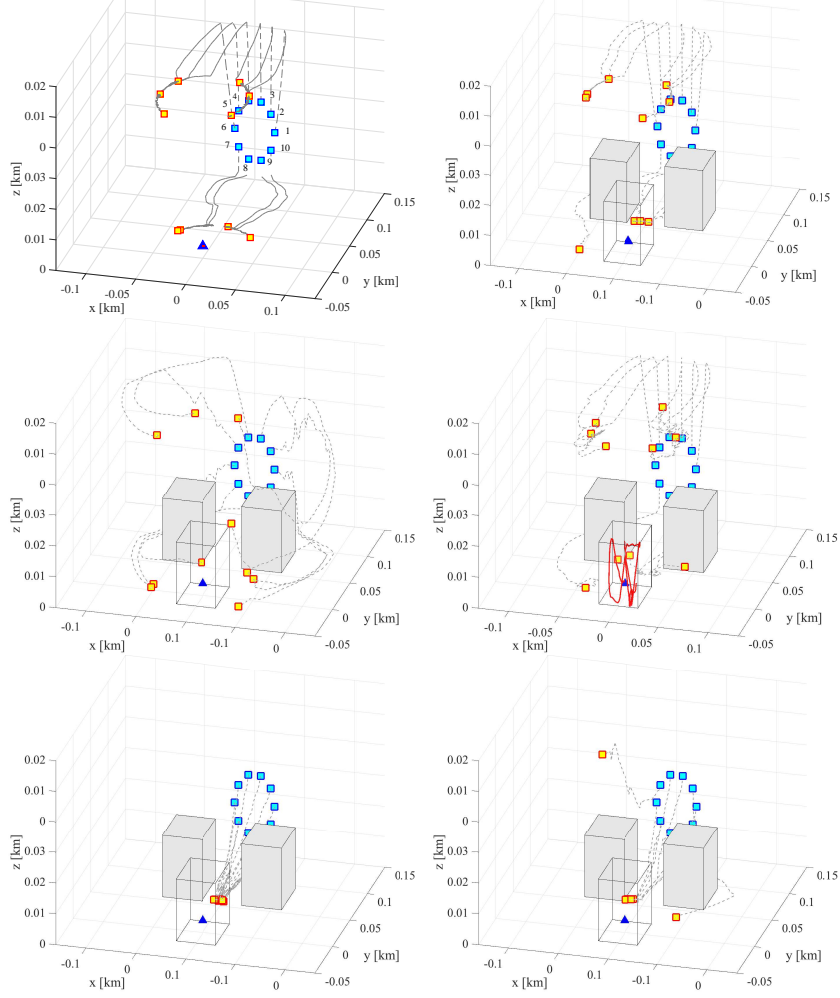

Fig. 2. Example of UAV trajectories obtained with the A-optimality criterion. Top-left: $\mathcal{N}=\mathcal{N}_{\mathrm{r}}$, LOS scenario; Top-Right: $\mathcal{N}=\mathcal{N}_{\mathrm{r}}$, NLOS scenario; Middle-left: $\mathcal{N}=\mathcal{N}_{\mathrm{b}}$; Middle-right: $\mathcal{N}=\mathcal{N}_{\mathrm{r}}$, with a dynamic source; Bottom-left: $\mathcal{N}=\mathcal{N}_{\mathrm{j}}$; Bottom-right: $\mathcal{N}=\mathcal{N}_{\mathrm{r}} \cup \mathcal{N}_{\mathrm{b}} \cup \mathcal{N}_{\mathrm{j}}$.

for NLOS [17], and $\sigma_{\mathrm{b}, 0}=10^{\circ}$ [16]. The safety distances were $d_{\mathrm{U}}^{*}=1 \mathrm{~m}, d_{\mathrm{S}}^{*}=50 \mathrm{~m}$ and $d_{\mathrm{O}}^{*}=5 \mathrm{~m}$. The range of altitudes of the UAVs was set to $\left[z_{\min }, z_{\max }\right]=[2,25] \mathrm{m}$. There were 100 Monte Carlo trials where at each iteration a different measurement noise was generated, with $N=10$, a communication range of $d_{\text {hop }}=100 \mathrm{~m}$, and $h_{\max }=1$. Each Monte Carlo simulation was restricted to 550 steps. In the heterogeneous case, we set $\mathcal{N}_{\mathrm{r}}=\{1,4,7,10\}, \mathcal{N}_{\mathrm{b}}=\{2,5,8\}$ and $\mathcal{N}_{\mathrm{j}}=\{3,6,9\}$ with the numbering reported in Fig. 2-topleft. The initial positions of UAVs was set along an ellipse in the $X Z$-plane of radii $r_{\mathrm{x}}=20 \mathrm{~m}, r_{\mathrm{z}}=5 \mathrm{~m}$ and centered at $[0,150,8] \mathrm{m}$.

In Fig. 2, the UAV positions are displayed as squares of different colors (for time instants $k=\{0,550\}$ ), the source position is the blue triangle. The trajectories are drawn as dashed lines, and the obstacles creating NLOS situations are the grey parallelepipeds. Figure 2 displays some qualitative examples of trajectories estimated using the A-optimality criterion. More specifically, we considered four cases depending on the information collected by the UAVs:

- The ranging-only case, when $\mathcal{N}=\mathcal{N}_{\mathrm{r}}$, as in Fig. 2-top;

- The bearing-only case, when $\mathcal{N}=\mathcal{N}_{\mathrm{b}}$, as in Fig. 2 . middle-left;

- The joint ranging and bearing case, when $\mathcal{N}=\mathcal{N}_{\mathrm{j}}$, as in Fig. 2.bottom-left; 


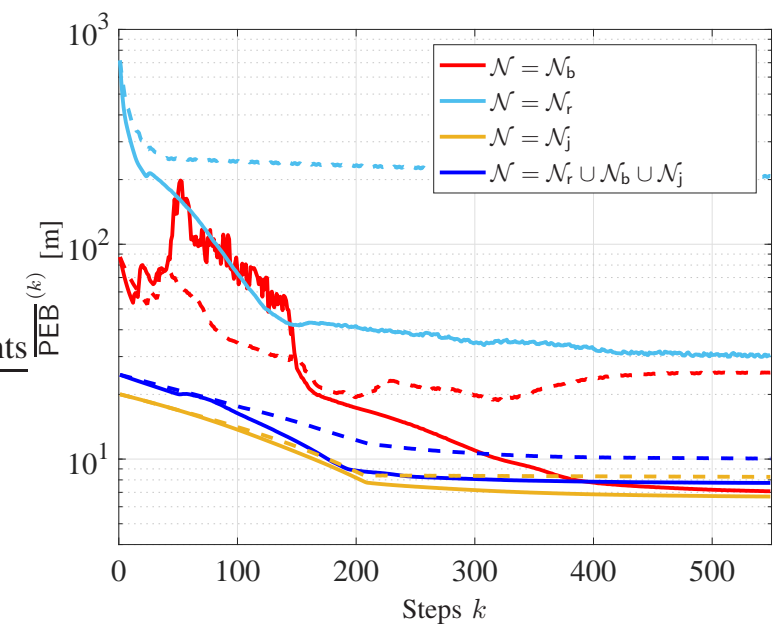

Fig. 3. PEB vs. type of measurements with $N=10, d_{\text {hop }}=100 \mathrm{~m}$ and $h_{\max }=1$. The dashed lines refer to the D-optimality criterion whereas the continuous lines to the A-optimality approach.

- The case with heterogeneous UAVs, i.e., $\mathcal{N}=\mathcal{N}_{\mathrm{r}} \cup \mathcal{N}_{\mathrm{b}} \cup$ $\mathcal{N}_{\mathrm{j}}$, as in Fig. 2-bottom-right.

In all the simulations, the source was supposed to be static. Anyway, in Fig. 2.-middle-right, an example with a dynamic source is reported. As can be noticed, due to the fact that the movements are constrained by the perimeter, there are no significant differences compared to Fig. 22-top-right.

To assess the localization accuracy, we considered the PEB averaged over the Monte Carlo trials, defined as

$$
\overline{\mathrm{PEB}}^{(k)}=\frac{1}{N \cdot N_{\mathrm{MC}}} \sum_{i=1}^{N} \sum_{m=1}^{N_{\mathrm{MC}}} \sqrt{\operatorname{tr}\left(\mathbf{J}^{-1}\left(\mathbf{p}_{0} ; \mathbf{q}_{i, m}^{(k)}\right)\right)},
$$

where $\mathbf{q}_{i, m}^{(k)}$ represents the set of UAVs positions available at the $i$ th UAV at time instant $k$ at the $m$ th Monte Carlo iteration.

We will show that when the swarm is composed of heterogeneous UAVs, the performance in terms of localization accuracy is similar to that obtainable with UAVs acquiring both ranging and bearing information.

In Fig. 3 the PEB is presented as a function of the criterion used for the navigation (i.e., A- and D-optimality) and considering different UAV sensing capabilities. As can be observed, when the UAVs have bearing-only measurements, in NLOS settings, obstacle obstructions lead to a singular FIM, which might prevent the UAVs from further navigation. The step behaviour of the PEB curve in the bearing-only case is due to this effect. Namely, before becoming a singular matrix, the FIM decreases (and, hence, the PEB increases) because some UAVs have already entered the NLOS area and their measurements are no longer related with the source position. To avoid that the UAVs stop due to a non-sufficient number of informative measurements, a "random" approach is adopted where the UAVs move randomly along the last followed direction. On the contrary, for the ranging-only case, the effect of the NLOS is an increased ranging error variance. These issues are solved when the UAVs can obtain both ranging and bearing measurements or when the network is composed of heterogeneous UAVs. An interesting point emerging from Fig. 3 is that, for navigation in NLOS environments, the Aoptimality criterion outperforms the D-optimality in terms of localization accuracy for most of the navigation time. In fact, when using the D-optimality criterion, the UAVs are trapped in the NLOS areas longer than when the A-optimality is used.

\section{CONCLUSIONS}

In this paper, we proposed a distributed network of heterogeneous UAVs whose navigation task is to minimize the localization error of a static source. In particular, the proposed control law aims at minimizing two Fisher information-driven cost functions (i.e., A- and D-optimality functions) in a distributed fashion and at each time instant. The cost functions were derived under the assumption of having access to bearing and/or ranging estimates. The results demonstrate that multimodal UAVs adopting the A-optimality criterion achieve the best localization performance for the considered scenario.

\section{REFERENCES}

[1] R. J. Wood, "The first takeoff of a biologically inspired at-scale robotic insect," IEEE Trans. Robot., vol. 24, no. 2, pp. 341-347, 2008.

[2] V. Kumar and N. Michael, "Opportunities and challenges with autonomous micro aerial vehicles," The Int. J. Robot. Research, vol. 31, no. 11, pp. 1279-1291, 2012.

[3] H. Bayerlein, P. De Kerret, and D. Gesbert, "Trajectory optimization for autonomous flying base station via reinforcement learning," in Proc. 2018 19th Int. Workshop Signal Process. Adv. Wireless Commun. (SPAWC). IEEE, 2018, pp. 1-5.

[4] S. Zhang et al., "Location-aware formation control in swarm navigation," in Proc. 2015 Globecom Workshops. IEEE, 2015, pp. 1-6.

[5] A. Guerra et al., "Collaborative target-localization and informationbased control in networks of UAVs," in Proc. 19th Int. Workshop Signal Process. Adv. Wireless Commun. (SPAWC). IEEE, 2018, pp. 1-5.

[6] A. Guerra, D. Dardari, and P. M. Djurić, "Joint indoor localization and navigation of uavs for network formation control," in Proc. 2018 52nd Asilomar Conf. Signals, Sys., and Comput. IEEE, 2018, pp. 13-19.

[7] C. Goerzen, Z. Kong, and B. Mettler, "A survey of motion planning algorithms from the perspective of autonomous UAV guidance," J. Intell. Robotic Syst., vol. 57, no. 1-4, p. 65, 2010.

[8] S. Ragi and E. K. Chong, "UAV path planning in a dynamic environment via partially observable Markov decision process," IEEE Trans. Aerosp. Electron. Syst., vol. 49, no. 4, pp. 2397-2412, 2013.

[9] Z. M. Kassas and T. E. Humphreys, "Receding horizon trajectory optimization in opportunistic navigation environments," IEEE Trans. Aerosp. Electron. Syst., vol. 51, no. 2, pp. 866-877, 2015.

[10] D. Ucinski, Optimal measurement methods for distributed parameter system identification. CRC Press, 2004.

[11] K. Dogancay, "UAV path planning for passive emitter localization," IEEE Trans. Aerosp. Electron. Syst., vol. 48, no. 2, pp. 1150-1166, 2012.

[12] E. Tzoreff and A. J. Weiss, "Path design for best emitter location using two mobile sensors," IEEE Trans. Signal Process., vol. 65, no. 19, pp. 5249-5261, 2017.

[13] S. A. A. Shahidian and H. Soltanizadeh, "Optimal trajectories for two UAVs in localization of multiple RF sources," Trans. Institute Meas. Control, vol. 38, no. 8, pp. 908-916, 2016.

[14] Z. Kassas and T. E. Humphreys, "Motion planning for optimal information gathering in opportunistic navigation systems," in AIAA Guidance, Navig., Control (GNC) Conf., 2013, p. 4551.

[15] D. Dardari, E. Falletti, and M. Luise, Satellite and terrestrial radio positioning techniques: a signal processing perspective. Elsevier, 2012.

[16] J. T. Isaacs et al., "Quadrotor control for RF source localization and tracking," in Proc. 2014 Int. Conf. Unmanned Aircraft Sys. (ICUAS). IEEE, 2014, pp. 244-252.

[17] S. Gezici, "A survey on wireless position estimation," Wireless personal communications, vol. 44, no. 3, pp. 263-282, 2008. 
[18] M. R. Gholami, R. M. Vaghefi, and E. G. Ström, "RSS-based sensor localization in the presence of unknown channel parameters," IEEE Trans. Signal Process., vol. 61, no. 15, pp. 3752-3759, 2013.

[19] P. M. Djurić and P. Closas, "On self-assessment of proficiency of autonomous systems," in Proc. 2019 Int. Conf. Acoustics, Speech and Signal Process. (ICASSP). IEEE, 2019, pp. 5072-5076.

[20] S. M. Kay et al., Fundamentals of statistical signal processing, Vol. I: Estimation theory. Prentice Hall Upper Saddle River, NJ, USA:, 1998.

[21] D. G. Luenberger, Y. Ye et al., Linear and nonlinear programming. Springer, 1984, vol. 2. 